\title{
Nanoscale
}

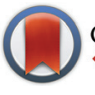

CrossMark

\&lick for updates

Cite this: Nanoscale, 2015, 7, 92

Received 17th September 2014,

Accepted 2nd November 2014

DOI: $10.1039 / c 4 n r 05426 a$

www.rsc.org/nanoscale

\section{Ultra-long zinc oxide nanowires and boron doping based on ionic liquid assisted thermal chemical vapor deposition growth $\uparrow$}

\author{
Andreas Menzel, ${ }^{a}$ Kris Komin, ${ }^{a}$ Yang Yang, $\star^{a}{ }^{a}$ Firat Güder, ${ }^{a}$ Vanessa Trouillet, ${ }^{b}$ \\ Peter Werner ${ }^{\mathrm{C}}$ and Margit Zacharias ${ }^{\mathrm{a}}$
}

Ionic liquid assisted growth of ultra-long $\mathrm{ZnO}$ nanowires from thermal chemical vapor deposition and the incorporation of dopants into the $\mathrm{ZnO}$ lattice have been investigated. We find that decomposed components of the ionic liquid at higher temperatures facilitate ultra-long vapor-liquid-solid $\mathrm{ZnO}$ nanowires that exhibit an unusual a-axis orientation. In particular, the ionic liquid $\mathrm{BMImBF}_{4}$ has been studied and the mechanism of the nanowire growth model in response to the use of the ionic liquid has been explained. We show that boron which is part of the investigated ionic liquid incorporates into the $\mathrm{ZnO}$ lattice and serves as a donor source. Electrical measurements were conducted and have shown an enhanced electrical conductivity $(\rho=0.09 \Omega \mathrm{cm})$ when using the ionic liquid assisted growth approach. This work represents a step towards the controlled doping for designing future nanowire devices.

During the last two decades, much effort has been focused on the growth and properties of nanowires (NWs) of different material systems which could serve as excellent building blocks for nanosized future electronic and optoelectronic devices. ${ }^{1-3}$ ZnO nanowires are one of the most studied materials among the different materials that are used in the nanowire community. A number of key parameters can influence the growth of metal oxide nanowires by thermal chemical vapor deposition (CVD) as was discussed and schematically demonstrated before. ${ }^{4}$ Many groups have studied the various effects of certain parameters and obtained different shapes of nanostructures. Controlled conditions including vacuum tight

\footnotetext{
${ }^{a}$ Laboratory for Nanotechnology, Department of Microsystems Engineering - IMTEK, University of Freiburg, Freiburg 79110, Germany.

E-mail: andreas.menzel@imtek.uni-freiburg.de

${ }^{b}$ Institute for Applied Materials (IAM) and Karlsruhe Nano Micro Facility (KNMF), Karlsruhe Institute of Technology (KIT), Eggenstein-Leopoldshafen, Germany ${ }^{c}$ Max Planck Institute of Microstructure Physics, Weinberg 2, D-06120 Halle, Germany

$\dagger$ Electronic supplementary information (ESI) available. See DOI: 10.1039/ c4nro5426a

\$Current address: State Key Laboratory of Materials-Oriented Chemical Engineering, College of Chemistry and Chemical Engineering, Nanjing Tech University, Nanjing 210009, China.
}

tube furnaces as a precondition for reliable experiments are needed for deeper understanding of the various growth modes and forms. In our previous work, we derived several shape diagrams that determine the parameter regions in which particular nanostructures can be obtained. ${ }^{4-6}$ Typical growth modes are the vapor-solid (VS) and the vapor-liquid-solid (VLS) nanowires. However, mixed modes forming thicker nanorods, nanobelts, or nanosheets, and even the suppression of growth are also observed for certain parameter combinations of source-substrate temperature. ${ }^{6}$ Also the effects of carrier gas and reaction gas were studied thoroughly in order to evaluate the transport mechanism of the evaporated $\mathrm{Zn}$ growth species and the region where $\mathrm{ZnO}$ nanowires can be grown. ${ }^{5}$

In a previous work, we demonstrated that the addition of an ionic liquid (IL) into the thermal CVD system can change the growth mode of $\mathrm{ZnO}$ nanowires from VS to VLS and also the growth orientation from $c$ to $a$-orientation. ${ }^{7}$ However, the growth mechanism was not fully understood which motivated us to conduct a more detailed investigation in order to get a better understanding of the role of the ionic liquid that assists the CVD growth of $\mathrm{ZnO}$ nanowires. Ionic liquids are in general a special class of salts that consist mostly of organic cations and inorganic anions and remain liquid even at room temperature. ${ }^{8}$ The changed growth mechanism of nanowires when the ionic liquid is present was attributed to a carbon assisted and self-catalytic growth of the $\mathrm{ZnO}$ nanowires. It was suggested that growth orientation change is caused by the large number of carbon that is released during the decomposition of the IL, but also due to the presence of $\mathrm{F}$ and $\mathrm{N}$ atoms (from $\mathrm{BMImBF}_{4}$ ) which may change the polarity and thus the growth orientation of the nanowire. ${ }^{7}$ The presence of carbon was confirmed by photoluminescence (PL) measurements that revealed a reduced defect related emission while annealing led to a strongly enhanced defect emission as a result of the removal of carbon passivation during the annealing process in air. $^{7}$ Moreover, such carbon passivation of $a$-oriented nanowires was also responsible for a reduced CO sensing performance as compared to polar ZnO VS nanowires. ${ }^{9}$ However, there was no experimental verification to prove the presence of 
$\mathrm{F}$ and $\mathrm{N}$ within the nanowires. This motivated us to conduct much deeper investigations to find the reason for the switching between $a$ and $c$-orientations.

To date, the role of ionic liquids in the growth of metal oxide nanowires by an ionic liquid assisted thermal CVD approach remains rather unclear. We will focus in this study on the effects which the additional IL source has on the nanowire growth. Due to the nature of the $\mathrm{BMImBF}_{4}$ compound, the IL can also act as a dopant supplier which was not considered in previous studies. We propose that boron which is part of the IL can be incorporated into the $\mathrm{ZnO}$ nanowires during the CVD growth and will play a major role in the tremendous change of the growth behavior beside carbon assisting the growth process. Electrical measurements as well as X-ray photoelectron spectroscopy (XPS) spectral analysis will be used to support this hypothesis.

ZnO nanowires were synthesized via a carbo-thermal CVD approach using a horizontal 1 inch diameter 1-zone tube CVD furnace as shown in Fig. 1a. The growth is conducted under laminar low flow conditions enabling an upstream deposition mode (against the carrier gas flow direction) where the $\mathrm{ZnO}$ source boat ( $\mathrm{ZnO}$ and $\mathrm{C}$ powder mixture) is placed in the center of the tube. The IL source $\left(25 \mu \mathrm{L} \mathrm{BMImBF}_{4}\right.$ dropped on a $\mathrm{Si}$ piece) is placed next to the $\mathrm{ZnO}$ source boat in the upstream direction. An Si (100) sample with a $5 \mathrm{~nm}$ Au layer acting as the catalyst is placed at a position where a temperature of $800{ }^{\circ} \mathrm{C}$ is valid. During the experiment, an $\mathrm{O}_{2}$ flow rate of $0.001 \mathrm{sccm}$ and a relatively low Ar flow rate of $7 \mathrm{sccm}$ were used at a chamber pressure of 200 mbar. In our experiment, the growth occurs in an upstream deposition mode because the diffusion effect of the $\mathrm{Zn}$ growth species has a significant influence due to the rather low flow rate of the carrier gas flow as simulated before. ${ }^{5}$ Several experiments were conducted to estimate the growth rate. The $\mathrm{ZnO}$ nanowire samples were investigated by HRSEM imaging and XPS measurements. XPS investigation of the nanowire samples was performed in a K-Alpha spectrometer (ThermoFisher Scientific, East Grinstead, UK) using a microfocused, monochromated $\mathrm{Al} \mathrm{K \alpha}$ $\mathrm{X}$-ray source (400 $\mu \mathrm{m}$ spot size). Data acquisition and processing using the Thermo Avantage software is described elsewhere. ${ }^{10}$ All spectra were referenced to the $\mathrm{C}$ 1s peak $(\mathrm{C}-\mathrm{C}, \mathrm{C}-\mathrm{H})$ at $285.0 \mathrm{eV}$ binding energy controlled by means of the well known photoelectron peaks of metallic $\mathrm{Cu}, \mathrm{Ag}$, and $\mathrm{Au}$. Electrical measurements from single $\mathrm{ZnO}$ nanowires were done using four-point-probe configurations. Electrical contacts were made using a direct-laser writing system which is a photolithographic approach and yields a reliable and cost effective contact formation for nanowires (as compared to the standard e-beam lithographic techniques). The IL based $\mathrm{ZnO}$ nanowires were scratched from the Si substrate and dispersed using acetone solution and spread on a $\mathrm{SiO}_{2}$ substrate. Subsequently after drying, a photoresist (AZ5214E) was spin-coated on the dispersed nanowire sample and baked. The four-probe contacts were made using a direct-laser writing system ( $\mu$ PG-101 from НЕMT) to expose the photolithographic defined contact areas. A $250 \mathrm{~nm}$ thick Al layer was deposited after development by a metal evaporator and the lift-off in acetone solution was conducted to obtain the single wire based device. In order to remove the remnant carbon on the $\mathrm{ZnO}$ nanowire, an $\mathrm{O}_{2}$ plasma treatment was conducted. A microprobe station that is connected to a device analyzer (Agilent B1500A) was used to extract the electrical parameters of the $\mathrm{ZnO}$ nanowire.

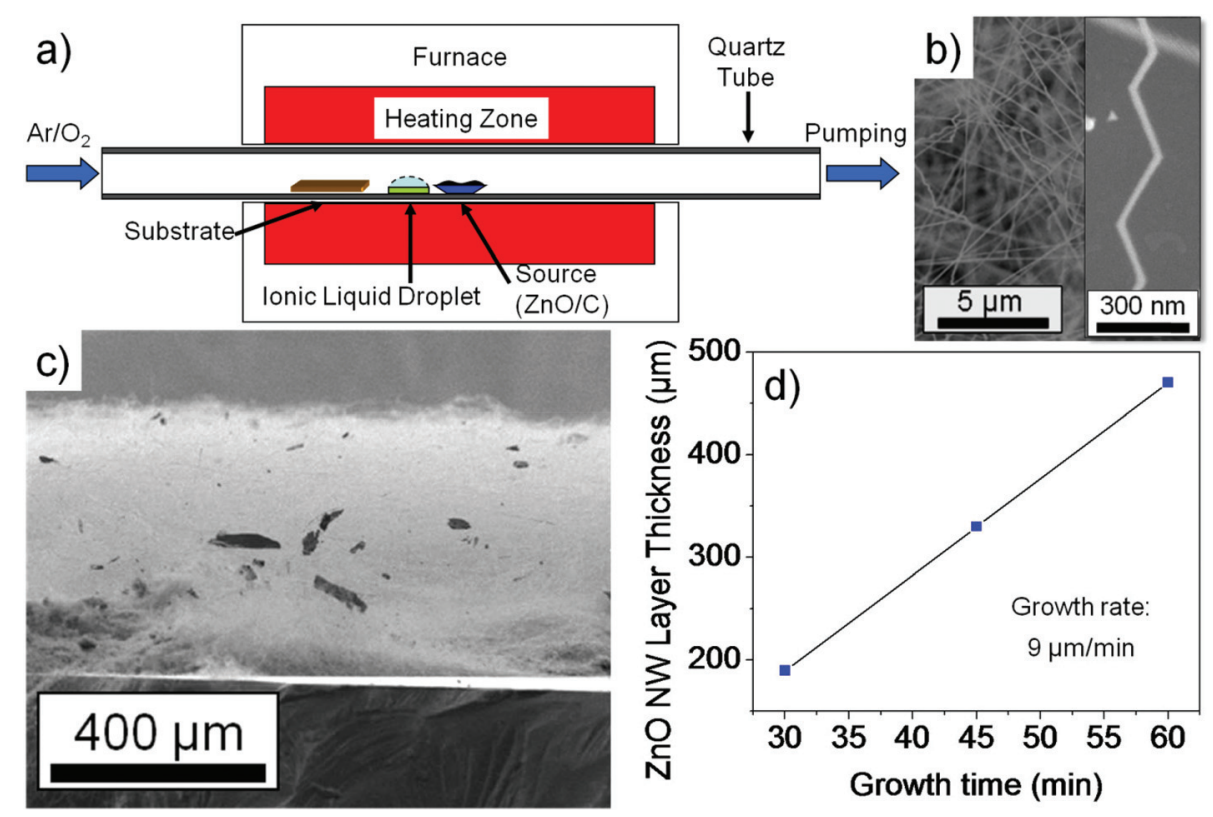

Fig. 1 (a) Schematic diagram of the one-zone (1 in. quartz tube) thermal CVD setup to grow ZnO nanowires by an IL assisted approach. The IL droplet is placed in the upstream direction from the $\mathrm{ZnO} / \mathrm{C}$ powder source. (b) SEM micrograph of the a-axis VLS ZnO nanowires. The inset shows the kinking from the a-axis nanowire growth. (c) Cross-sectioned SEM micrograph of IL ZnO nanowires. (d) Plot of ZnO nanowire layer thickness versus the growth time to determine the growth rate. 
Passivation of the nanowire sample surface was carried out by conformal coating with a $20 \mathrm{~nm}$ thick $\mathrm{Al}_{2} \mathrm{O}_{3}$ layer by a low temperature $\left(115{ }^{\circ} \mathrm{C}\right)$ process using atomic layer deposition (ALD). Please note that the alumina was deposited onto the nanowires that were already contacted by the metal. The electrical measurements were conducted before and after the $\mathrm{Al}_{2} \mathrm{O}_{3}$ deposition.

In recent studies we demonstrated that the $\mathrm{ZnO}$ nanowire growth follows mainly a VS nanowire growth when only a $\mathrm{ZnO}+\mathrm{C}$ source is used. A VLS growth is only observed in a rather specific parameter window (e.g. higher temperatures and specific $\mathrm{Ar}$ and $\mathrm{O}_{2}$ environments). ${ }^{4,6}$ However, controlled switching between the slow VS growth and the fast VLS growth is possible by adding the additional IL source into the growth chamber. To reverse this process to a VS growth a cleaning procedure of the CVD quartz tube reactor with $\mathrm{HCl}$ is needed. Fig. 1b shows randomly oriented nanowires and nanobelts grown on the Au covered $\mathrm{Si}$ substrate. In contrast to experiments without the IL, the nanowires are grown over almost the entire substrate area $(1.5 \mathrm{~cm} \times 2.5 \mathrm{~cm})$, i.e. the temperature gradient effects (of up to $150 \mathrm{~K}$ in our case) do not seem to play a major role. Also, as seen in the cross-sectional SEM image in Fig. 1c, a highly dense $\mathrm{ZnO}$ nanowire film with a thickness of up to $\sim 450 \mu \mathrm{m}$ is observed after only 1 hour growth. With a $\mathrm{ZnO}$ nanowire film growth rate of $9 \mu \mathrm{m} \mathrm{min} \mathrm{m}^{-1}$ this high-throughput nanowire growth is interesting for industrial applications, preparing papers or mats based on $\mathrm{ZnO}$ nanowires which could be important for medical applications. It is also very important to note that a homogeneous growth area for $\mathrm{ZnO}$ nanowires extends over a length of $\sim 7 \mathrm{~cm}(1$ inch tube furnace) which represents in our system a temperature range of $\sim 800{ }^{\circ} \mathrm{C}$ to $950{ }^{\circ} \mathrm{C}$ (in the upstream direction). Since the nanowires based on the thin Au catalyst film grow in random interwoven orientations as indicated in Fig. 1b, we conclude from the respective observations that the nanowires have lengths of several millimeters or even centimeters as indicated in Fig. 1c. A top view of the SEM image of a ZnO nanowire sample that was grown within a time of 30 minutes can be seen in Fig. S1 in the ESI. $\dagger$ At the edge of this sample the nanowires show lengths of several hundred $\mu \mathrm{m}($ e.g. $>200 \mu \mathrm{m})$ to demonstrate that the ionic liquid assisted nanowire growth can lead to superlong nanowires. Due to the very long length, the low diameters of around $50 \mathrm{~nm}$, and the 'wool' like structure it is rather difficult to exactly determine the length of the nanowires by SEM imaging, and therefore only estimations were made. We conducted experiments with different growth times in order to estimate the growth rate of the interwoven $\mathrm{ZnO}$ nanowire film. From Fig. 1d, an astonishingly tremendous $\mathrm{ZnO}$ nanowire film growth rate in the range of $9 \mu \mathrm{m}$ $\min ^{-1}$ was estimated that suggests an extremely high yield of nanowires using this IL assisted approach.

An experiment with a growth time of only 5 min was conducted on Au dots deposited on a GaN (0001)/sapphire substrate to investigate the early growth state of the nanostructure. Fig. $2 \mathrm{~b}$ shows the nanostructure of an area with nanowires. There are Au dots typical for VLS growth observed at the tips of such nanowires. In a previous study, we had already confirmed the $\mathrm{Au}$ dot on the tip of the nanowires through high-resolution transmission electron microscopy (HRTEM) investigations which indicates that we have a catalyst assisted VLS nanowire growth. ${ }^{7}$ The associated electron diffraction pattern (also demonstrated previously) prove the growth of the nanowires along the $a$-axis. Another indication that the nanowires or nanobelts grow along the $a$-axis is the kinked or zigzagged growth as seen in Fig. 1b (inset). Such kinking behavior can originate from a thermal or strain instability at the liquidsolid interface because the free energy of equivalent $\{11 \overline{2} 0\}$ planes is the same. ${ }^{11,12}$ We have also investigated the crystallographic behavior of the kinked $\mathrm{ZnO}$ nanowires using TEM. A bright-field TEM image that represents a kinked $\mathrm{ZnO}$ nanowire by this ionic liquid assisted approach is shown in Fig. S2 (ESI $\dagger$ ). In our TEM investigations we found that the entire nanowire structure is single crystalline and no dislocations or defects can be identified at the joints and boundaries. Hence, we can conclude that the nanowire growth is single crystalline and follows a VLS mechanism along the $a$-axis, opposite to the normally preferred $c$-axis growth. At this stage, the question arises as to why the $\mathrm{ZnO}$ nanowires change the growth mode, the growth orientation, and so drastically the growth rate as compared to the nanowire growth without the assistance of an ionic liquid.

For a deeper understanding we have to consider the composition of the used IL $\mathrm{BMImBF}_{4}$ that contains besides hydrocarbon compounds elements like N, F, and B, which may play an important role, too. Ionic liquids are in general stable salts in the liquid phase but when approaching higher temperatures above $500{ }^{\circ} \mathrm{C}$ they thermally decompose into smaller components. To our knowledge there exist no studies that investigate the decomposition of IL at temperatures such as $800{ }^{\circ} \mathrm{C}$ and higher so that we can only speculate about the behavior of such a complex system. Studies from pyrolysis-gas chromatography experiments by Ohtani et al. have shown that $\mathrm{BMImBF}_{4}$ mainly decomposes at higher temperatures $\left(\sim 550{ }^{\circ} \mathrm{C}\right)$ by the cleavage of $\mathrm{C}-\mathrm{N}$ and $\mathrm{C}-\mathrm{C}$ bonds into alkyl chains but also 2 -fluoropropane components were detected. ${ }^{8}$ Please note that there exist no studies so far that investigate the behavior at higher temperatures as the mentioned reference. Interestingly, no peak that originates from the boron compound appeared in their studies. We conducted XPS observations to investigate the composition of the IL assisted nanowires: no peaks that contain $\mathrm{F}$ or $\mathrm{N}$ components were observed which can be explained probably due to the detection limit of the XPS setup. However, we were able to identify a certain amount of B concentration ( 0.9 at $\%$, see also Fig. 4 a, upper curve) in an oxidized state. Obviously the presence of $\mathrm{B}$ atoms in the $\mathrm{ZnO}$ nanowire is comparatively higher than that of $\mathrm{F}$ and $\mathrm{N}$. Hence, $\mathrm{B}$ incorporation into the $\mathrm{ZnO}$ nanowire affects the nanowire growth performance besides the carbon assisted growth. Additionally, we would like to suggest at this point that B substitutes $\mathrm{Zn}$ lattice sites. It is known that carbon can be used to assist the growth of nanowires and enhance the growth rate (not as much as we see here) but no switching in the growth 

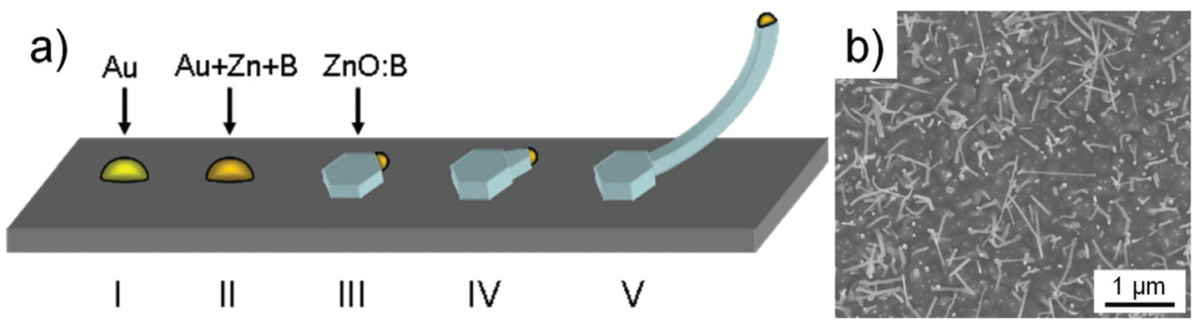

Fig. 2 (a) Proposed growth process of the ZnO nanowires during the IL assisted VLS growth route. (b) Top view of ZnO nanowires on a GaN (1000) on the sapphire substrate using Au as a catalyst.

orientation was observed to our knowledge. ${ }^{13,14}$ We have also conducted a reference experiment where we grew VS ZnO nanowires without using the IL to confirm that B originates from the additional IL source. The XPS result (lower curve in Fig. 4a) did not show any B related peak.

A study by Fan et al. investigated the growth of $\mathrm{ZnO}$ nanobelts as a response to indium dopants that appear from carbothermal evaporation of the $\operatorname{In}_{2} \mathrm{O}_{3} / \mathrm{C}$ system. A similar switch from $c$ to $a$-orientation was observed during their study that was attributed to the incorporation of In atoms into the $\mathrm{ZnO}$ crystal. ${ }^{11,12}$ The generated $\mathrm{Zn}$ and In vapor transported to the substrate nucleates at the catalyst and forms a ternary phase (Au-Zn-In) which supersaturates in the liquid alloy droplet. Saturation and strain rise and change the surface free energy and the nanowire is layer by layer pushed out of the $\mathrm{ZnO}: \mathrm{In}$ nuclei. ${ }^{11,12}$ In this case the precipitation and oxidation of this VLS nanowire growth is now different and the $a$-oriented nanowires grow instead of the $c$-oriented. We now adapt this model to our case as described in Fig. 2a when boron is evaporated during the decomposition of $\mathrm{BMImBF}_{4}$ (please note, $\mathrm{B}$ and In are both group III elements). Here, similar to the VLS mechanism $\mathrm{ZnO}$ is formed between the liquid-solid interface but the additional boron incorporation yields the formation of a $\mathrm{ZnO}: \mathrm{B}$ nucleus with a nanowire that grows out of the side facets of the Au-Zn-B alloy particle. The formation of zigzag or kinked nanowires is a further characteristic that shows similarities to the In-assisted nanowire growth as seen in Fig. 1b (inset) and in the previously reported TEM observations. ${ }^{7}$

Moreover, similar to the In case, the B impurities represent an n-type dopant source for the nanowires and can be utilized

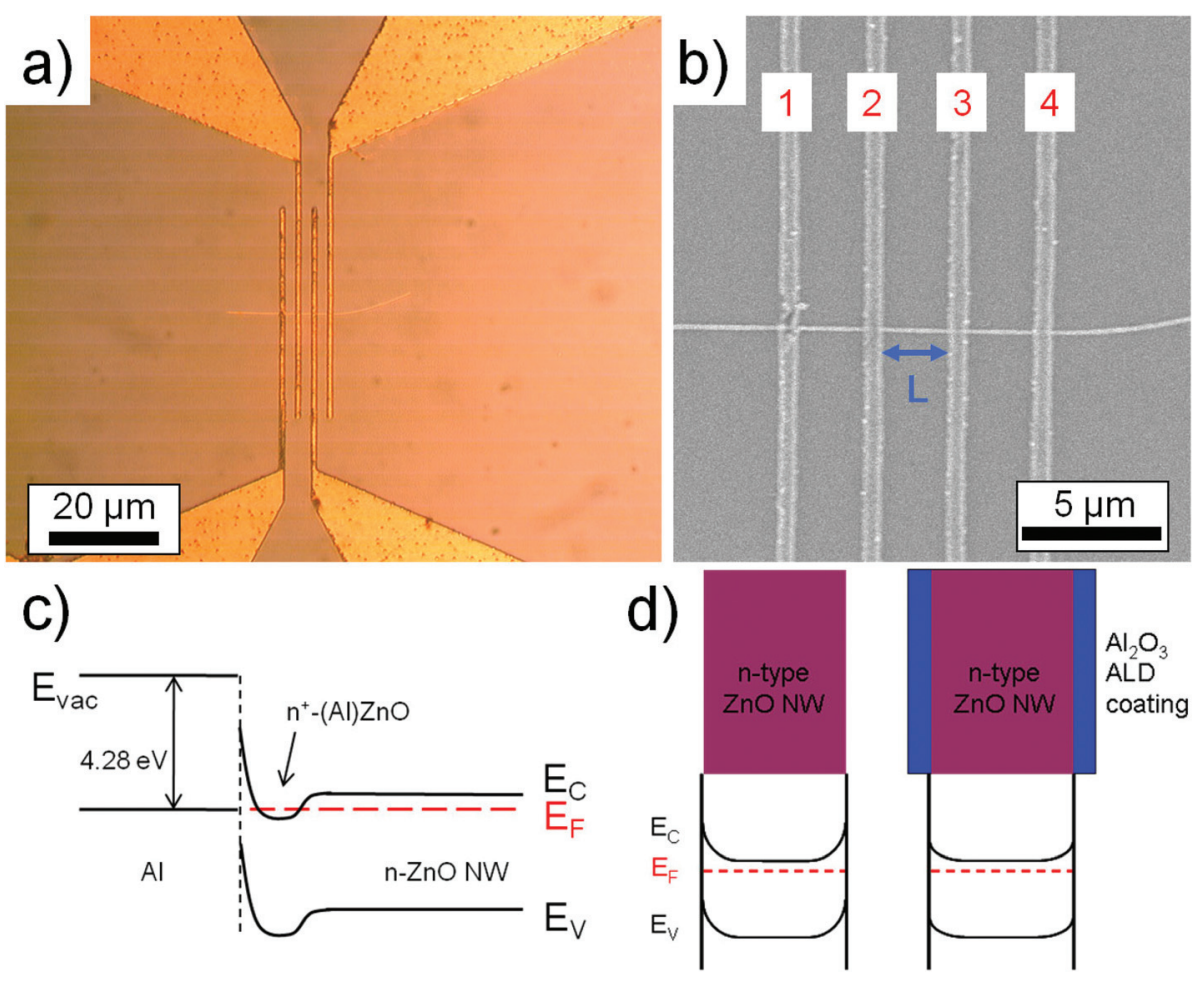

Fig. 3 (a) Optical microscope image of a 4-probe contacted IL ZnO nanowire using Al as metal contacts. (b) SEM micrograph that shows the IL ZnO nanowire and for contact terminals to determine the resistivity. (c) Schematic band diagram of an Al-based ohmic contact on a $\mathrm{ZnO}$ nanowire. (d) Band diagrams of IL ZnO nanowires: (left) non-passivated nanowires, and (right) passivation with a $20 \mathrm{~nm} \mathrm{Al}_{2} \mathrm{O}_{3}$ layer by $\mathrm{ALD}$. $E_{\mathrm{V}}$ and $E_{\mathrm{C}}$ represent the valence and conduction band energies and $E_{\mathrm{F}}$ represents the Fermi level. 
to enhance the electrical conductivity of the material when employed as an FET device. Hence, four-point-probe contacts were created (see Fig. 3a and b) using $\mathrm{Al}$ as the metal to form ohmic contacts between the metal and the ZnO n-type semiconductor. ${ }^{15,16}$ It was shown by Kim et al. that $\mathrm{Al}$ atoms at the $\mathrm{Al}-\mathrm{ZnO}$ interface diffuse into the $\mathrm{ZnO}$ surface region, forming an increased doping concentration of $\mathrm{ZnO}$ such that $\mathrm{n}^{+}$-(Al) $\mathrm{ZnO}$ results (see also the schematic energy band diagram in Fig. 3c). ${ }^{16} E_{\mathrm{V}}$ and $E_{\mathrm{C}}$ represent the valence and conduction band energies, respectively, and $E_{\mathrm{F}}$ represents the Fermi level. Consequently, the barrier width at the $\mathrm{Al}-\mathrm{ZnO}$ interface is reduced such that a tunneling process through this barrier is enhanced. An electrical resistivity of $\rho=0.4 \Omega \mathrm{cm}$ was extracted from the four-point $I-V$ curve in Fig. $4 \mathrm{~b}$ for bare nonpassivated $\mathrm{ZnO}$ nanowires. The $\mathrm{ZnO}$ nanowires have a certain amount of interface states on the surface that cause a
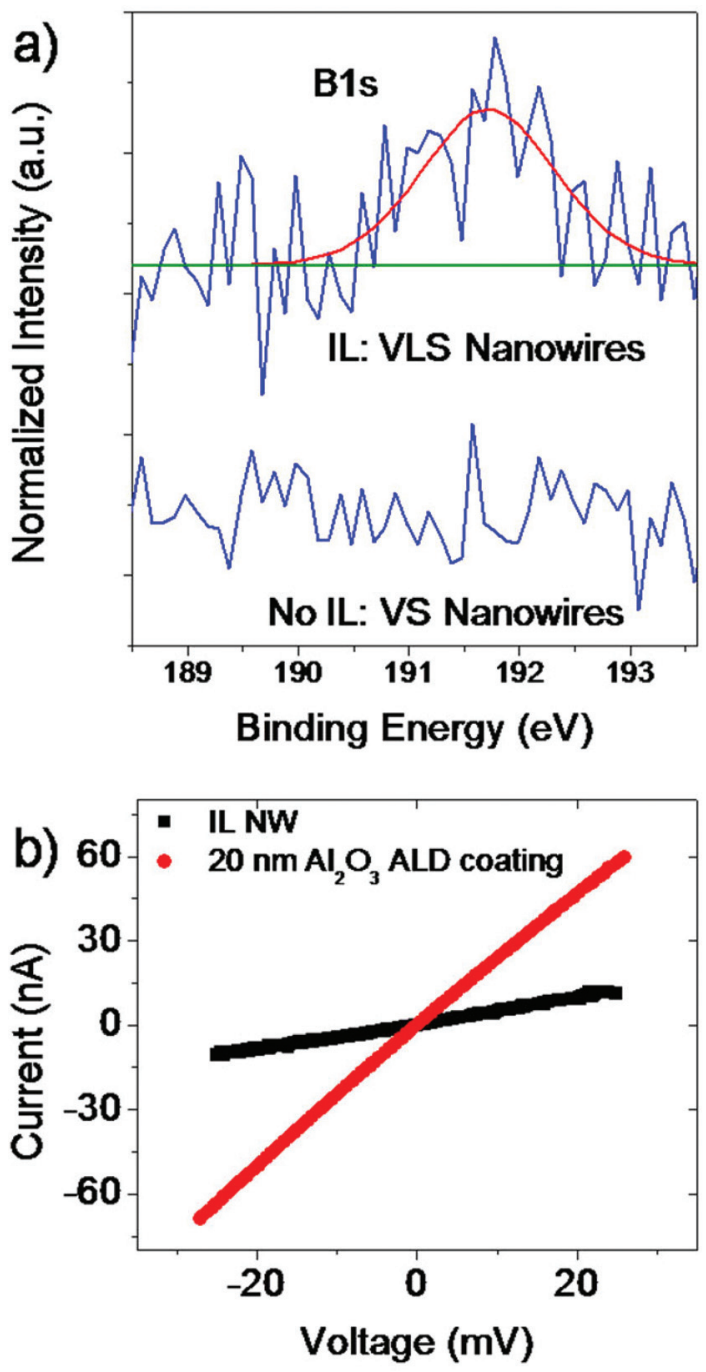

Fig. 4 (a) XPS spectrum of IL ZnO nanowires. The lower spectrum represents reference experiments with pure VS nanowires (no IL used). (b) Electrical measurements of non-passivated $(\rho=0.4 \Omega \mathrm{cm})$ and with $20 \mathrm{~nm} \mathrm{Al}_{2} \mathrm{O}_{3}$ passivated $(\rho=0.09 \Omega \mathrm{cm}$ ) IL ZnO nanowires. depletion of charge carriers and also donor deactivation as schematically shown in the energy band diagram in Fig. 3d (left). ${ }^{17,18}$ If we now passivate the surface of these contacted nanowire samples with a conformal $20 \mathrm{~nm}$ coating of $\mathrm{Al}_{2} \mathrm{O}_{3}$, we reduce the amount of interface states and defects and reduce the surface depletion such that more donors are activated, as indicated in the band diagram in Fig. 3d (right). ${ }^{19}$ The now extracted electrical resistivity from passivated samples based on the $I-V$ curve in Fig. $4 \mathrm{~b}$ is $\rho=0.09 \Omega \mathrm{cm}$, i.e. one order of magnitude less. The value is much lower than that typically obtained from VS nanowires obtained without any additional impurity source $(\rho \gg 4 \Omega \mathrm{cm}) \cdot{ }^{20-22}$ Hence, it can be suggested that the increased value in electrical conductivity is originated by the assisted use of an ionic liquid during the nanowire growth that influences besides the morphology also the electrical properties of the $\mathrm{ZnO}$ nanowires.

In conclusion, in contrast to previously reported studies, the $\mathrm{BMImBF}_{4} \mathrm{IL}$ assisted nanowire growth utilizes mainly the components carbon and boron where boron is significantly involved in the change of the VLS nanowire growth characteristics as compared to the pure VLS ZnO nanowire growth. We discussed the two main effects that affect the $\mathrm{ZnO}$ nanowire growth compared to pure nanowire growth without an IL: first is that B incorporates into the $\mathrm{ZnO}$ lattice and induces the switching in growth orientation to $a$-orientation and second, the carbon that originates from the IL decomposition additionally catalyzes/enhances the nanowire growth resulting in a significantly faster growth. Both effects enable a very fast $\mathrm{ZnO}$ nanowire layer growth rate which was determined to be at least $9 \mu \mathrm{m} \mathrm{min}^{-1}$. Since B is an n-type source for the II/VI ZnO material, we have shown that the IL assisted growth can be used to incorporate donors. These assumptions were supported by XPS and electrical measurements of the $\mathrm{ZnO}$ nanowires.

\section{Acknowledgements}

This work was supported by the German Research Foundation (DFG) under the contract ZA 191/24-1 (Freiburg).

\section{References}

1 Y. Cui and C. M. Lieber, Science, 2001, 291, 851-853.

2 V. Schmidt, H. Riel, S. Senz, S. Karg, W. Riess and U. Gösele, Small, 2006, 2, 85-88.

3 A. Menzel, K. Subannajui, F. Güder, D. Moser and O. Paul, Adv. Funct. Mater., 2011, 21, 4342-4348.

4 A. Menzel, K. Subannajui, R. Bakhda, Y. Wang, R. Thomann and M. Zacharias, J. Phys. Chem. Lett., 2012, 3, 2815-2821.

5 A. Menzel, R. Goldberg, G. Burshtein, V. Lumelsky, K. Subannajui, M. Zacharias and Y. Lifshitz, J. Phys. Chem. C, 2012, 116, 5524-5530. 
6 C. Wongchoosuk, K. Subannajui, A. Menzel, I. A. Burshtein, S. Tamir, Y. Lifshitz and M. Zacharias, J. Phys. Chem. C, 2011, 115, 757-761.

7 N. S. Ramgir, K. Subannajui, Y. Yang, R. Grimm, R. Michiels and M. Zacharias, J. Phys. Chem. C, 2010, 114, 10323-10329.

8 H. Ohtani, S. Ishimura and M. Kumai, Anal. Sci., 2008, 24, 1335-1340.

9 K. Subannajui, C. Wongchoosuk, N. S. Ramgir, C. Wang, Y. Yang, A. Hartel, V. Cimalla and M. Zacharias, J. Appl. Phys., 2012, 112, 034311.

10 K. L. Parry, A. G. Shard, R. D. Short, R. G. White, J. D. Whittle and A. Wright, Surf. Interface Anal., 2006, 38, 1497-1504.

11 H. J. Fan, B. Fuhrmann, R. Scholz, C. Himcinschi, A. Berger, H. Leipner, A. Dadgar, A. Krost, S. Christiansen, U. Gösele and M. Zacharias, Nanotechnology, 2006, 17, S231-S239.

12 H. J. Fan, A. S. Barnard and M. Zacharias, Appl. Phys. Lett., 2007, 90, 143116.

13 T. Yanagida, A. Marcu, H. Matsui, K. Nagashima, K. Oka, K. Yokota, M. Taniguchi and T. Kawai, J. Phys. Chem. C, 2008, 112, 18923-18926.
14 C. Cheng, T. L. Wong, W. Li, C. Zhu, S. Xu, L. Wang, K. K. Fung and N. Wang, AIP Adv., 2011, 1, 032104032104.

15 L. J. Brillson and Y. Lu, J. Appl. Phys., 2011, 109, 121301.

16 K. H. Kim, K. Kim, S. Park, T. Seong and I. Adesida, J. Appl. Phys., 2003, 94, 4225.

17 M. T. Björk, H. Schmid, J. Knoch, H. Riel and W. Riess, Nat. Nanotechnol., 2009, 4, 103-107.

18 A. Casadei, P. Krogstrup, M. Heiss, J. A. Röhr, C. Colombo, T. Ruelle, S. Upadhyay, C. B. Sorensen, J. Nygard and A. Fontcuberta i Morral, Appl. Phys. Lett., 2013, 102, 013117.

19 J.-P. Richters, T. Voss, D. S. Kim, R. Scholz and M. Zacharias, Nanotechnology, 2008, 19, 305202.

20 S. N. Cha, J. E. Jang, Y. Choi, G. A. J. Amaratunga, G. W. Ho, M. E. Welland, D. G. Hasko, D.-J. Kang and J. M. Kim, Appl. Phys. Lett., 2006, 89, 263102.

21 S. N. Cha, B. G. Song, J. E. Jang, J. E. Jung, I. T. Han, J. H. Ha, J. P. Hong, D. J. Kang and J. M. Kim, Nanotechnology, 2008, 19, 235601.

22 J. Goldberger, D. J. Sirbuly, M. Law and P. Yang, J. Phys. Chem. B, 2005, 109, 9-14. 